\title{
Enriching Medical Students' Attitudes About Child and Adolescent Psychiatry Through Viewing of "Starless Dreams" in a Cross-Cultural Seminar on Adverse Childhood Experiences
}

\author{
Krishna Patel $^{1} \cdot$ Esha Bansal $^{1} \cdot$ Yonis Hassan $^{1} \cdot$ Susan Kim $^{1} \cdot$ Arifa Zaidi $^{1} \cdot$ Timothy Rice $^{1}$ (D) \\ Received: 14 May 2021 / Accepted: 6 January 2022 / Published online: 16 February 2022 \\ (C) Academic Psychiatry 2022
}

Across the USA [1] and globally [2], undergraduate medical education in child and adolescent psychiatry (CAP) is limited. Efforts to promote child and adolescent psychiatry generally focus at the post-graduate level, though proposals for strengthening undergraduate medical education in CAP exist [3]. Cinema offers creative opportunities for psychiatric education [3], and several authors [4-6] have already explored the use of film to teach CAP and human development [7]. Outcome data concerning the use of film remains sparse. Considering that pre-clerkship exposure is an important factor for medical students pursuing psychiatric training [8] and that evening-hour education has documented benefits of being free from workday time pressures [9], we aimed to study the capabilities of an evening cinema program for early-career medical students in relation to CAP training and recruitment. The COVID-19 pandemic and resulting in-person activity restrictions raise the need for virtual medical education interventions. Additionally, increased contemporary interest in diversity and social justice heighten the importance of education in global and cross-cultural settings that are not readily available in-person.

By creating opportunities to collectively consider human behaviors and circumstances, film screening and discussion may model thoughtful consideration of clinical concepts and the diverse lived experiences of patients' mental illness [10]. Specifically, narrative-based learning through film may contribute to the formation of well-rounded clinicians by "preparing medical students to engage in the shared decision-making process with their patients" [11] and augmenting narrative competence, or "the capacity to recognize, absorb, metabolize, interpret, and be moved by stories of illness" [12].

Timothy Rice

Timothy.rice@mountsinai.org

Icahn School of Medicine at Mount Sinai, New York, NY, USA
Film-based learning approaches may also mitigate existing challenges of motivating students to pursue advanced CAP training. Since many CAP patients are a vulnerable population, direct medical student involvement in patient care may be restricted by confidentiality and sensitivity concerns. Exposure may also be limited by CAP divisional or departmental sizes at many medical schools. Connecting students through film with post-graduate CAP trainees and faculty can provide a conduit for ongoing mentorship. For these reasons, film-based interventions show promise.

\section{Starless Dreams: a Teaching Tool for Child and Adolescent Psychiatry}

The 2017 film Starless Dreams by Mehrdad Oskouei weaves together multiple themes relevant to CAP including childhood trauma, gender and sexual violence, and the interrelationships of crime and poverty. Set in a correctional facility at the outskirts of Tehran, Oskouei's acclaimed film centers on seven adolescent women. Through serial personalized interview, Oskouei explores their lives before prison, their present-day realities, and their thoughts about the future. The film also captures their profound sense of community and camaraderie fostered by a shared understanding of one another's narratives and traumas. Over the film's course, each protagonist describes her childhood and the crimes that led to her incarceration; the young women's narratives include themes of early-life physical and sexual abuse, poverty, child marriage, drug addiction, and homelessness. Oskouei also documents the day-to-day lives of the incarcerated teenagers, including holiday celebrations, moments of personal grief, interactions with law enforcement and religious authority, collective recreational activities, and family visitations.

The protagonists of Starless Dreams recount narratives of gender violence that resonate with women's lived experiences 
across multiple sociopolitical periods in modern Iran's history. The 2002 film Women's Prison (Zendān-e Zanān), directed by Manijeh Hekmat through decades of fieldwork with incarcerated women in Tehran, had previously depicted the status of female prisoners in Iranian society at three timepoints: (1) the post-revolution conflict with Iraq (1984); (2) the economic liberalization and political authoritarianism of the Ransanjani regime (1992); and (3) during the religious-progressive tensions following the Khatami reformation (2001) [13]. Women's Prison had painted a narrative picture in which prisoners' experiences of physical and sexual abuse by husbands and fathers are further compounded by homelessness, crime, substance use, and trafficking; however, prisoners also reclaimed power through acts of political resistance and alliances with other women. In a review of the film, critic Izzat al-Sadat Gushahgir noted that adolescent prisoners across these periods have facilitated more hopeful dialogue around societal change: "In contemporary Iran, where women struggle to fight and negate the innumerable mechanisms of oppression in order to survive... a teenage prisoner represents a symbolic figure of hope, of dreaming of a new generation who will create drastic changes in the society" [14].

Starless Dreams enters here. The film can be an effective teaching tool to communicate key principles of CAP such as adverse childhood experiences (ACEs) and stimulate discussion on the interplay between broader issues like prisons and mental health, the intergenerational transmission of trauma, recovery, and resilience. While several films have been used to teach about post-traumatic stress disorder $[15,16]$ and loss and bereavement [17], only one [16] has pertained to youth trauma.

In our study, we wished to assess whether (1) participants emerged with a greater subjective understanding of childhood trauma and (2) the combination of film screening and open discussion can increase interest in and appreciation of psychiatric practice, with indirect implications for recruitment into CAP. Relatedly, we also aimed to assess the benefits and limitations of film as a means of instruction.

\section{Setting and Intervention Details}

The film discussion of Starless Dreams was a seventy-five-minute virtual meeting held on April 21, 2021, as part of an eight-session, voluntary, not-for-credit course entitled "Film in Medicine" at the Icahn School of Medicine at Mount Sinai. On April 15, all enrolled students were notified of the discussion via multiple emails. All students indicating interest were invited along with their guests to view the film and participate in the discussion.

The session occurred via a virtual medium during the COVID-19 pandemic and was facilitated by a CAP faculty member (TR) and a third-year postgraduate resident in psychiatry pursuing fellowship training in child and adolescent psychiatry (AZ). The session began with a ten-minute introduction to Oskouei's inspiration for the film and an overview of discussion themes including Oskouei's interviewing style, cross-cultural depictions of adverse childhood experiences, and the confluence of child marriage, addiction, homelessness, intergenerational violence, crime, poverty, and unemployment in shaping trauma experiences.

After discussing the effectiveness and ethics of Oskouei's interviewing style, the two facilitators focused on a series of clinical correlates local to the medical school. The facilitators discussed similarities and differences between each of the female protagonists' stories and patients and families they had encountered in clinical practice. Participants were also led to calculate ACE scores for one of the women and to discuss fundamental psychological principles including learned helplessness and neurobiological principles including hypothalamic-pituitary-adrenal (HPA) axis dysregulation of post-traumatic stress disorder. The facilitators encouraged the participants to freely comment and ask questions throughout the discussion to encourage a collaborative, organic learning environment.

At the end of the discussion, all medical student session participants were invited to complete an anonymous nine-question survey gauging their satisfaction, changes in their understanding of child development, and perceptions of psychiatry. This survey was one of eight administered at the conclusion of each session of the Icahn School of Medicine at Mount Sinai "Film in Medicine" elective course; the Institutional Review Board determined the study to be eligible for exempt status. The survey consisted of Likert-type questions to allow for quantitative evaluation and open-ended questions for qualitative evaluation. Likert-type questions were based on the following five-point scale: "Strongly Disagree; Disagree; Neutral; Agree; Strongly Agree".

\section{Quantitative and Qualitative Outcomes}

Quantitative and qualitative feedback was very positive and supported both hypotheses pertaining to the potential of film discussion to increase participants' understanding of childhood trauma and interest in psychiatry. All survey questions and data, including all responses to each question, are available upon request.

Four of five medical students were present for the entire film discussion and completed the anonymous survey. All four respondents self-reported an increased understanding of general mental health, with two reporting "strong agreement" and the other two reporting "agreement". Participants self-reported an average understanding of $7 / 10$ before the week's film screening and discussion and an average of $8 / 10$ afterwards when asked: "Before/after watching this week's 
film and participating in today's discussion, I would rate my understanding of this week's theme [childhood trauma] on a scale of $1-10$ as $(0=$ no understanding, $5=$ average or layperson understanding, $10=$ expert understanding)".

Likewise, though only one of four respondents indicated the film and discussion changed their view of psychiatrists in general (the other three reported "neutral"), all four reported that they came away with more favorable impressions of psychiatrists and the field of psychiatry. Three out four respondents agreed with the sentiments that they were "more interested in learning about the field of psychiatry" and "more interested in a career in psychiatry."

When specifically asked about changes in perception about psychiatry, one respondent wrote: "It made me more sure that I want to go into psychiatry." In addition, multiple responses suggest that connecting film scenes to clinical correlates concurrently improve understanding and stimulate interest. One response stated, "It was great to hear about Dr. Rice's experiences in his training and practice and about emotional/mental support required". A separate participant connected the social trauma inflicted upon the protagonists resonated to a teaching point from the CAP faculty member concerning the benefits of asking adolescents directly about suicidal ideation:

"learning from Dr. Rice (when he said that talking about something will not necessarily lead to someone doing something... or that manipulation/social relations [can] leave more of a mark on someone's life than other types of trauma)."

Session attendance also evoked critical internal reflections about psychiatric practice and the field of psychiatry. Survey responses suggest that these reflections may be informed by participants' prior personal or clinical experiences and framed constructively in terms of future professional development. For instance, one attendee said:

"I learned about how psychiatrists perceive certain wording ("suicide", etc.) and how they try to destigmatize it...but I still think there is more to be said about destigmatizing vs desensitization, which are two behaviors that are seen commonly in psychiatry (speaking from personal experience). The line between them is very thin and I would have liked to have discussed that more (...through my learning more about the field's more altruistic efforts, my perception has changed for the "better")."

These positive qualitative accounts are consistent with the quantitative data detailed above. Furthermore, emergent themes from responses to open-ended survey questions and perspectives shared during the class discussion also support the utility of Starless Dreams in exploring cross-cultural depictions of adverse childhood experiences and prompting reflection on the extent to which psychiatric care can adequately treat patients with deeply-rooted trauma. As one respondent wrote, "The challenges of being a psychiatrist, particularly in our very imperfect system, weighed heavy on our discussion."

Numerous scenes from Starless Dreams motivate discussion around how child and adolescent psychiatrists can help children who experience multiple ACEs, such as the youth protagonists of the film. Child psychotherapy can facilitate children and adolescents to build resilience in the service of interrupting unprocessed intergenerational cycles of trauma $[18,19]$. The community between the young women offers opportunity for discussion of how social support promotes resilience [18], while the presence of a CAP within the discussion can emphasize how clinicians can strengthen resilience in children and adolescents through psychotherapy [19]. Because the young women in the film vividly discuss their past, present their present, and dream of the future, the concept of the intergenerational transmission of trauma can be powerfully taught alongside the potential of CAPs to intervene in strength-based approaches. The women's depictions of parental addiction and other parental challenges provide an avenue to discuss how care for the family can provide care for the child; the opportunities afforded through a child psychiatrists' presence and voice within a team-based approach may be underscored. These discussions can strengthen medical students and post-graduate residents' interest in CAP careers.

Findings are limited in their generalizability. Results were cross-sectional and based on a small sample size. Therefore, findings are best interpreted tentatively. Future studies ought to utilize more robust study designs. As participation in the course and session was voluntary, the offering likely self-selected for students with a pre-existing interest in psychiatry. However, these could also be positive features if the smaller discussion group and common interest inadvertently contributed to a more comfortable group dynamic. Film also has elaborated limitations in teaching about psychopathology [20], although the documentary quality with non-fictitious adolescents in Starless Dreams may overcome some of the inherent distortions in this medium. Finally, questions in the post-discussion survey, which was the primary means of outcome assessment, were not specific for CAP. For instance, many questions refer to "psychiatry/psychiatrists" in general but not child and adolescent mental health care. This limitation was a product of use of an identical survey for all sessions presented across the course, which provided simplicity to facilitate participant response for the study of the course in its entirety.

Overall, predominantly positive quantitative and qualitative feedback, combined with the relative ease and 
convenience of hosting film screenings and discussions, highlights the promise of films like Starless Dreams to convey multicultural aspects of child and adolescent development.

Acknowledgements We wish to thank the Nexus Learning program at the Icahn School of Medicine at Mount Sinai, which promotes student and faculty engagement in a diverse range of courses. Engagement in this manuscript was stimulated through activity within a Nexus elective entitled "Film and Medicine." We additionally wish to thank all the students who participated in our elective.

\section{Declarations}

Ethical Considerations This manuscript reports on results of a study that was determined to be exempt human research as defined by DHHS regulations 45 CFR 46.101(b)(1).

Disclosures On behalf of all authors, the corresponding author states that there is no conflict of interest.

\section{References}

1. Sawyer MG, Giesen F, Walter G. Child psychiatry curricula in undergraduate medical education. J Am Acad Child Adolesc Psychiatry. 2008 Feb 1;47(2):139-47.

2. Mian AI, Milavić G, Skokauskas N. Child and adolescent psychiatry training: a global perspective. Child Adolesc Psychiatr Clin N Am. 2015 Oct 1;24(4):699-714.

3. Martin A, Bloch M, Pruett K, Stubbe D, Belitsky R, Ebert M, Leckman JF. From too little too late to early and often: child psychiatry education during medical school (and before and after). Child Adolesc Psychiatr Clin N Am. 2007;16:17-43.

4. Kaye DL, Ets-Hokin E. The Breakfast Club: utilizing popular film to teach adolescent development. Acad Psychiatry. 2000 Jun;24(2): $110-6$.

5. Schlozman SC. A unique tool for teaching development: a review of Dr. Geri Fox's 20 years of family video material created to teach human development. Acad Psychiatry. 2012 May 1;36(3):256-7.
6. Guerrero APS. An approach to finding teaching moments on families and child development in Disney films. Acad Psychiatry. 2015;39:225-30.

7. Schlozman SC. Why psychiatric education needs the humanities. Acad Psychiatry. 2017;41:703-6.

8. Lau T, Zamani D, Lee EK, Asli KD, Gill J, Brager N, et al. Factors affecting recruitment into psychiatry: a Canadian experience. Acad Psychiatry. 2015;39:246-52.

9. Brenner AM, Beresin EV, Coverdale JH, Louie AK, Balon R, Guerrero APS, Roberts LW. Time to teach: addressing the pressure on faculty time for education. Acad Psychiatry. 2018;42:5-10.

10. Webster CR, Valentine LC, Gabbard GO. Film clubs in psychiatric education: the hidden curriculum. Acad Psychiatry. 2015;39:6014.

11. Milota MM, van Thiel GJMW, van Delden JJM. Narrative medicine as a medical education tool: a systematic review. Medical Teacher. 2019;41:802-10.

12. Charon R. What to do with stories: the sciences of narrative medicine. Can Fam Physician. 2007 Aug 1;53(8):1265-7.

13. Gushahgir IA. Women's prison. J Middle East Women's Stud. 2006;2(1):138-40.

14. Hekmat M. Women's Prison. Iran: Bamdad Film; 2002.

15. Shapiro P, Tobia A, Aziz R. Is the film Unbreakable really about PTSD with dissociation? Acad Psychiatry. 2018;42:871-2.

16. Ramos LD, Guimarães FS, Ventriglio A, de Andrade AG, Bhugra D, Lotufo-Neto F, Castaldelli-Maia JM. DSM-5 post-traumatic stress disorder criteria in "Precious" (2009): media content analysis for educational purposes. Acad Psychiatry. 2017;41:396-404.

17. Furst BA. Bowlby goes to the movies: film as a teaching tool for issues of bereavement, mourning, and grief in medical education. Acad Psychiatry. 2007;31:407-10.

18. Woods-Jaeger BA, Cho B, Sexton CC, Slagel L, Goggin K. Promoting resilience: breaking the intergenerational cycle of adverse childhood experiences. Health Educ Behav. 2018 Oct;45 (5):772-80.

19. Prout TA, Malone A, Rice T, Hoffman L. Resilience, defense mechanisms, and implicit emotion regulation in psychodynamic child psychotherapy. J Contemp Psychother: On the Cutting Edge of Modern Developments in Psychotherapy. 2019;49(4):235-44.

20. Ramchandani $\mathrm{D}$. The downside of teaching psychopathology with film. Acad Psychiatry. 2012 Mar;36(2):154-5.

Publisher's Note Springer Nature remains neutral with regard to jurisdictional claims in published maps and institutional affiliations. 